UB-ECM-PF 94/9

March, 1994

\title{
Renormalization-group improved effective potential for finite grand unified theories in curved spacetime
}

\author{
E. Elizalde 凹 \\ Center for Advanced Studies, C.S.I.C., Camí de Santa Bàrbara, 17300 Blanes, \\ and Department E.C.M. and I.F.A.E., Faculty of Physics, University of Barcelona, \\ Diagonal 647, 08028 Barcelona, Catalonia, Spain \\ and \\ S.D. ODINTSOVF \\ Tomsk Pedagogical Institute, 634041 Tomsk, Russia.
}

\begin{abstract}
The renormalization-group improved effective potential - to leading-log and in the linear curvature approximation - is constructed for "finite" theories in curved spacetime. It is not trivial and displays a quite interesting, exponential-like structure - in contrast with the case of flat spacetime where it coincides with the classical potential. Several possible cosmological applications, as curvature-induced phase transitions and modifications of the values of the gravitational and cosmological constants, are briefly discussed.
\end{abstract}

\footnotetext{
${ }^{1}$ E-mail: eli@zeta.ecm.ub.es, eli@ebubecm1.bitnet

${ }^{2}$ E-mail: odintsov@ebubecm1.bitnet. Also at the Department E.C.M., Faculty of Physics, University of Barcelona, Diagonal 647, 08028 Barcelona, Spain.
} 
1. Introduction. It is a well-known fact that supersymmetry (for an introduction, see [1]) leads to very interesting consequences. One of the most remarkable is the existence of finite theories (see, for instance, [2]-[四] and references therein). To be sure, supersymmetric theories are not the only ones which can be finite at the one-loop or two-loop level (see [4, 河), however, up to now only certain supersymmetric theories have been proven to be finite to all orders of perturbation theory ( $\mathrm{N}=4$ super Yang-Mills theory [6] is the best known example).

Different grand unified theories (GUTs) have been proposed which turn out to yield finite models. Some of them, as for instance the finite supersymmetric SU(5) GUT [7, may lead to quite reasonable phenomenological consequences and deserves careful attention as a realistic model of grand unification. Moreover, some generalizations of the concept of finite theory —as the asymptotically finite GUTs - have been proposed [8]. In such theories the zero charge problem is absent, both in the UF and in the IR limit, since in these limits the effective coupling constants tend to some constant values (corresponding to a finite phase).

When we consider a massless finite or massless asymptotically finite GUT there is no much sense in discussing quantum corrections to the classical potential in such a theory, since either they are simply absent or are highly suppressed asymptotically. However, when studying finite theories not in flat but in curved spacetime [0] (for a general review, see [10]) the situation changes drastically. There appears a non-trivial effective coupling constant $\xi(t)$ corresponding to the scalar-gravitational interaction which becomes important in different respects.

The purpose of this letter is to discuss the issue of the effective potential for finite (or asymptotically finite) theories in curved spacetime. More precisely, we will construct the renormalization-group $(\mathrm{RG})$ improved effective potential - in the linear curvature and leading-log approximation - for finite theories in curved spacetime. It will be shown that this potential has an interesting and unusual structure (as compared whith that for the standard theories). Among the possible cosmological applications of the model, curvature-induced phase transitions will be investigated. Some speculations about the associated variations of the cosmological and gravitational constants are made at the end.

2. The RG improved effective potential. Let us start from a certain multiplicativelyrenormalizable massless theory in curved spacetime. The general form for the Lagrangian of such kind of theory is

$$
L=\sqrt{-g}\left(L_{m}+L_{e x t}+\xi R \varphi^{2}\right),
$$

where $L_{m}$ corresponds to some GUT which includes multiplets of spinors $\psi$ and scalars $\varphi$, 
and gauge fields $A_{\mu}, \xi$ is the scalar-gravitational coupling constant, and

$$
L_{e x t}=a R^{2}+b G+c C_{\mu \nu \alpha \beta}^{2}+d \square R .
$$

Here $G$ is the Gauss-Bonnet invariant, $C_{\mu \nu \alpha \beta}$ the Weyl tensor and $R$ the curvature. Notice that the necessity to introduce $L_{\text {ext }}$ and $\xi R \varphi^{2}$ is demanded by renormalizability in curved spacetime (see [10 for an introduction).

We will consider the situation when $L_{m}$ corresponds to a finite (at least to the one-loop approximation) theory in flat space. Of course, in curved space such a theory is not finite, generally speaking, due to the extra terms in (11) (namely the vacuum energy and the nonminimal scalar-gravitational interaction term). This is why we prefer to call such a theory "finite", thus remarking that it is actually finite in flat space only. Finiteness means that the effective coupling constants of the theory have the following form

$$
g^{2}(t)=g^{2}, \quad h^{2}(t)=\kappa_{1} g^{2}, \quad \lambda(t)=\kappa_{2} g^{2}
$$

where $g^{2}, h^{2}$ and $\lambda$ are respectively the gauge, Yukawa and scalar couplings, $\kappa_{1}$ and $\kappa_{2}$ are some constants, and $g^{2}<<1$. Now we are going to study the RG improved effective potential [11] in such a theory (for a very recent discussion in the case of the standard model, together with a list of references, see [12]). In flat space such potential is given simply by a classical potential because there are no one-loop quantum corrections. In curved spacetime the situation changes a lot. For simplicity, let the classical potential contain only one scalar multiplet, i.e.

$$
V_{c l}=a \lambda \Phi^{4}-b \xi R \Phi^{2}
$$

where $a$ and $b$ are some positive constants. Then, by standard methods one can show that the RG improved effective potential in curved spacetime is given by [13]

$$
V=a \lambda(t) f^{4}(t) \varphi^{4}-b \xi(t) f^{2}(t) R \varphi^{2}
$$

where $t=(1 / 2) \ln \left(\varphi^{2} / \mu^{2}\right)$, and we have

$$
\begin{aligned}
\dot{\tilde{g}}(t) & =\bar{\beta}_{\widetilde{g}}(t), \widetilde{g}(0)=\widetilde{g} ; \quad \dot{\alpha}(t)=\bar{\delta}(t), \alpha(0)=\alpha ; \quad \dot{\xi}(t)=\bar{\beta}_{\xi}(t), \quad \xi(0)=\xi ; \\
f(t) & =\exp \left[-\int_{0}^{t} d t^{\prime} \bar{\gamma}\left(\widetilde{g}\left(t^{\prime}\right), \alpha\left(t^{\prime}\right)\right)\right],
\end{aligned}
$$

and $\left(\bar{\beta}_{\widetilde{g}}, \bar{\delta}, \bar{\beta}_{\xi}, \bar{\gamma}\right)=\left(\beta_{g}, \delta, \beta_{\xi}, \gamma\right) /(1+\gamma), \widetilde{g} \equiv\left\{g, h^{2}, \lambda\right\}, \alpha$ is the set of gauge parameters. For asymptotically free, as well as for non asymptotically free theories (such as the $\lambda \varphi^{4}$-theory) the RG improved effective potential (5) has been investigated in 13 already. Notice that 
this potential is obtained in the linear curvature approximation, which is good enough for GUTs corresponding to the curved spacetime of the early universe 14.

In "finite" theories, the effective coupling constants having analogues in flat spacetime are given by Eq. (3). The general structure of the one-loop effective coupling constant $\xi(t)$ for "finite" theories in curved spacetime has been already obtained in Ref. [9] as the solution of the RG equation $\dot{\xi}(t)=\beta_{\xi}(t)$, that is

$$
\dot{\xi}(t)=\left(\xi(t)-\frac{1}{6}\right) C g^{2}
$$

where the constant $C$ is fixed by specific features of the theory under discussion (it can be equal to zero for some theories). For $C \neq 0$, we have

$$
\xi(t)=\frac{1}{6}+\left(\xi-\frac{1}{6}\right) \exp \left(C g^{2} t\right)
$$

In particular, for the $\mathrm{SU}(2)$ finite gauge model of Ref. [4], we have $C=6$ or $C \simeq 28$ [9]. Hence (non-asymptotical conformal invariance), in such theories we have $|\xi(t)| \rightarrow \infty$ in the UF limit $(t \rightarrow \infty)$. In the models which have $C<0$ (examples of this kind of models can be found in [15, 5]), one obtains $\xi(t) \rightarrow 1 / 6$ (asymptotical conformal invariance).

Notice that our theory is defined not only in the UF limit but also has good behaviour in the IR limit (see (3)). In the IR limit the behaviour of $\xi(t)$ is reversed. In particular, for $C>0$ one has $\xi(t) \rightarrow 1 / 6$ as $t \rightarrow-\infty$ (asymptotical confomal invariance), with independence of the choice of initial value. It is interesting to observe that there exist models of inflationary universes (the variable Planck-mass models) for which the typical value of $\xi$ is very high, $|\xi| \sim 10^{4}$ [16] (for an introduction to the inflationary universe, see for instance [17]). The exponential running of $\xi(t)$ in "finite" GUTs (for models with $C<0$ ) provides a very natural way to obtain a very large $\xi$ in the early universe and, at the same time, a $\xi$ close to the conformal value at present energies. Hence, "finite" GUTs may provide a reasonable justification for the presence of such a large $\xi$ in the inflationary universe models of [16].

Now, in order to obtain the RG improved effective potential we should know the $\gamma$ function of the scalar field in (6). At the one-loop level, $\gamma \sim a_{1} g^{2}+a_{2} h^{2}$, where $a_{1}$ and $a_{2}$ are some constants whose value depend on the gauge choice and other features of the theory. As $h^{2}=\kappa_{1} g^{2}$, it turns out that $\gamma \sim\left(a_{1}+\kappa_{1} a_{2}\right) g^{2}$. Through the choice of gauge parameter, one can obtain different values for $\gamma$. As we restrict ourselves to finite theories, it seems rather reasonable to try to obtain as much finiteness in our theory as possible. Thus, we will choose the gauge in which the one-loop $\gamma$-function is equal to zero. This choice is 
always possible, moreover in supersymmetric finite theories it appears in a very natural way (specially if the superfield technique is used). Having done all this, it turns out that the RG improved effective potential (in the linear-curvature and leading-log approximation) for a "finite" theory in curved spacetime is given by

$$
V=a \kappa_{1} g^{2} \varphi^{4}-b \xi(t) R \varphi^{2}
$$

It is remarkable that this expression is valid for any $t$ (namely it is not restricted by perturbation theory), contrary to what happens in the standard situation [11]. One can consider its UF or IR limits (or regions close to those limits) at the same time.

If we choose to keep the gauge arbitrary, and do not demand that $\gamma$ vanishes, then we get

$$
V=a \kappa_{1} g^{2} f^{4}(t) \varphi^{4}-b \xi(t) f^{2}(t) R \varphi^{2}
$$

and $\gamma=C_{1} g^{2}, C_{1}$ being some constant which depends on the gauge parameter and on the features of the theory, $f(t)=\exp \left(-C_{1} g^{2} t\right)$, and $\xi(t)$ is given by (8) (since the corrections comming from the non-zero $\gamma$ in the equation $\dot{\xi}(t)=\beta_{\xi} /(1+\gamma)$ are of two-loop order).

Finally, for asymptotically finite theories [8] one can get

$$
V=a \lambda(t) f^{4}(t) \varphi^{4}-b \xi(t) f^{2}(t) R \varphi^{2}
$$

where we know only the asymptotic values of $\lambda(t), f(t)$ and $\xi(t)$ in the UF or IR limit, but ignore the behaviour of these effective couplings in the intermediate region. Recently, asymptotically finite theories have been used in [18] as the basis of a possible mechanism for quantum screening of the cosmological constant (this will be discussed below).

Hence, we have obtained a non-trivial RG improved effective potential for "finite" theories in curved spacetime. The most interesting property of this potential is the fact that it is valid, generally speaking, for any value of $t$ (provided, of course, that we are inside a region where perturbation theory has sense).

3. Curvature-induced phase transitions. It is common belief that the early universe has experienced one or several phase transitions. Such phase transitions could have been caused by modifications of some external parameters of the theory (as temperature, pressure or an external gravitational field). The inflationary phase of the early universe could probably have resulted from a phase transition of Coleman-Weinberg type. We will here discuss the possibility of a curvature-induced phase transition in a "finite" theory in curved spacetime, thus assuming as starting point that, in fact, the early universe can be described by a 
convenient "finite" GUT. As before, we shall work with the RG improved effective potential. The first realization of a gravitational phase transition, for the case of scalar QED in the De Sitter space, has been presented in Ref. [14] (for a review and a list of references, see [10]).

We will be interested in first order phase transitions, where the order parameter $\varphi$ experiences a quick change for some critical value, $R_{c}$, of the curvature (see, for example, [10]). As usually [14, 13, it is convenient to introduce the dimensionless parameters $x=\varphi^{2} / \mu^{2}$, $y=|R| / \mu^{2}$ and $\epsilon=\operatorname{sgn} R$, in terms of which the RG improved effective potential is given by (for simplicity, we consider the potential (91))

$$
\frac{V}{\mu^{4}}=a \kappa_{1} g^{2} x^{2}-b \in x y\left[\frac{1}{6}+\left(\xi-\frac{1}{6}\right) x^{C g^{2} / 2}\right] .
$$

The critical parameters corresponding to the first-order phase transition are defined by the standard conditions:

$$
V\left(x_{c}, y_{c}\right)=0,\left.\quad \frac{\partial V}{\partial x}\right|_{x_{c}, y_{c}}=0,\left.\quad \frac{\partial^{2} V}{\partial x^{2}}\right|_{x_{c}, y_{c}}>0 .
$$

The analysis of curvature-induced phase transitions, both for asymptotically free and for asymptotically non-free theories — using the corresponding RG improved effective potentialhas been carried out in Ref. [13].

For "finite" theories with the potential (9), the analysis of Eqs. (13) yields the following critical values for the phase transition

$$
x_{c}=\left[(1-6 \xi)\left(1-\frac{C g^{2}}{2}\right)\right]^{-2 / C g^{2}}, \quad \epsilon y_{c}=\frac{12 a \kappa_{1}}{b C}\left(-1+\frac{C g^{2}}{2}\right) x_{c} .
$$

In addition, we must restrict $a \kappa_{1} g^{2}\left(1-C g^{2} / 2\right)>0$ (this is the third condition in (13)) and also $\left|12 a \kappa_{1}\left(1-C g^{2} / 2\right) /(b C)\right|<1$ (otherwise the linear curvature approximation is broken).

Let us now produce some simple estimation of a possible result. Guided by the explicit

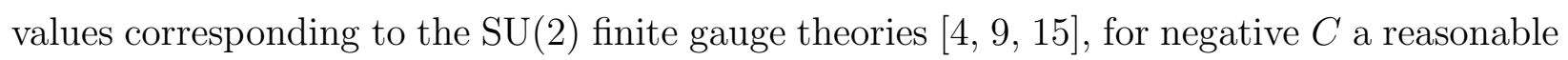
choice of parameters satisfying Eqs. (13) may be the following:

$$
b=\frac{1}{2}, \quad a=\frac{1}{4}, \quad \kappa_{1}=0.1, \quad C=-10 .
$$

Choosing moreover a small coupling constant $g^{2}=0.05$ and an initial value $\xi=0.15$, one gets

$$
x_{c}=2 \cdot 10^{-4}, \quad\left|y_{c}\right|=1.5 \cdot 10^{-5} \text {. }
$$

This estimation is already very much close to the standard value of the curvature in the GUT epoch, namely

$$
10^{-7} \leq|y| \leq 10^{-5}
$$


We have thus shown, in principle, the possibility of a gravitational phase transition for "finite" GUTs in curved spacetime. In fact this simple calculation hints very clearly towards the possibility of emergence of some kind of inflationary stage in the evolution of the early universe, based on the occurrence of a gravitational phase transition that, as we see, can appear quite naturally when we use a "finite" GUT to describe the early universe. Due to its simplicity, this is a rather remarkable result that deserves further study.

4. Induced cosmological and gravitational constants. It is a matter of fact that (at least in principle) in the assumed situation that the early universe can be described by some finite or asymptotically finite GUT, one can estimate the induced values of the cosmological and gravitational constants — or, more precisely, of their variations - by using the RG improved effective potential. Consider the RG improved effective potential under its most general form (10). By making the identifications

$$
G^{-1} \sim \xi(t) f^{2}(t) \varphi^{2}, \quad \Lambda \sim-\frac{g^{2} f^{2}(t) \varphi^{2}}{\xi(t)}
$$

one can actually estimate, in principle, the comological variations of the induced effective cosmological and Newton constants. In particular, by supposing [18] that a "finite" GUT phase of the early universe is appropriate from some energy scale $\mu_{U V}\left(t_{U V}\right.$ is large, i.e. the curvature is strong enough) to another scale $\mu_{I R}\left(t_{I R}\right.$ is small, i.e. the curvature is very weak), one can see that the variations of $G$ and $\Lambda$ are given by

$$
\frac{G_{I R}^{-1}}{G_{U V}^{-1}} \sim\left(\frac{\mu_{I R}}{\mu_{U V}}\right)^{\left(C-2 C_{1}\right) g^{2}}, \quad \frac{\Lambda_{I R}}{\Lambda_{U V}} \sim\left(\frac{\mu_{I R}}{\mu_{U V}}\right)^{-\left(C+2 C_{1}\right) g^{2}} .
$$

If one wants now to suppress the variation of the Newton constant, one can choose the gauge (and also, perhaps, the model) in order to obtain $C=2 C_{1}$. In this case an exponentially large screening of the cosmological constant appears which takes it from a very large value in the early universe to the extremely small one that it has at present energies. This simple mechanism, called RG screening of the cosmological constant, has been proposed in Ref. [18]. Unfortunately, the special choice that one has to do of the $\gamma$-function (or, in other words, of the constant $C_{1}$ ) is a kind of fine-tuning of the model. This feature, albeit common to almost any solution that has been proposed of the cosmological constant problem, is not very appealing.

In principle, the variations of $G$ and $\Lambda$ in "finite" GUTs depend very much on the choice of the model and gauge. In particular, for the 'natural' choice $C_{1}=0$ we get an exponential 
screening of $\Lambda$ but, at the same time, an exponential growth of the Newton constant. Such an outcome does not look very realistic either.

5. Conclusion. In this note we have studied in some detail the RG improved effective potential corresponding to massless "finite" GUTs in curved spacetime. Some cosmological speculations involving potentials of this kind have been analyzed. However, there is still a number of related questions that are left for further study. In particular, the important question of how a non-zero mass would influence the results of the RG potential. It is known that the answer to this question is not easy [12, 19], owing to the fact that usually one has simultaneously several effective masses in the theory and also because of the running of the vacuum energy, already in flat space. In curved space the situation is even more complicated and one is led to study the RG improved effective Lagrangian actually [20].

Another question concerns the generality of such a type of RG improved potential and if the results that we have obtained will be extrapolable (at least qualitatively) to other theories. To answer this, let us consider the $d=3$ abelian Chern-Simons gauge theory (for an inntroduction, see [21, 22]) interacting with matter. The interest of such kind of theories is mainly motivated by the possibility of their use as models of high-temperature superconductivity. The property of being finite is a very natural one in the case of an abelian Chern-Simons gauge theory [23]. Starting from a Chern-Simons theory with matter in a $d=3$ curved spacetime [24], one can construct the corresponding RG improved effective potential, what gives a similar result to that of Sect. 3, namely

$$
V=h(t) \Phi^{6}(t)-\xi(t) R \Phi^{2}(t)
$$

where $\Phi^{2}=\Phi^{*} \Phi, h$ is the scalar coupling constant, $h(t)=\kappa_{1} e^{4}$ (in the regime where the theory is finite), $e^{2}$ is the electromagnetic coupling constant, and $e^{2}(t)=e^{2}$ [23]. The effective field is given by $\Phi(t)=\exp \left(-C_{1} e^{4} t\right) \Phi$ and $\xi(t)=1 / 8+(\xi-1 / 8) \exp \left(C_{1} e^{4} t\right)$ [24. Hence, in the case of an abelian Chern-Simons theory with matter in curved spacetime, the RG improved effective potential has the same functional form as in the case of a "finite" GUT, and may also lead to $d=3$ curvature-induced phase transitions.

\section{Acknowledgments}

SDO would like to thank the members of the Dept. ECM, Barcelona University, for very kind hospitality. This work has been supported by DGICYT (Spain), project no. PB90-0022, and by CIRIT (Generalitat de Catalunya). 


\section{References}

[1] P. West, Introduction to supersymmetry and supergravity (World Scientific, Singapore, 1986).

[2] D.R.T. Jones and L. Mezinescu, Phys. Lett. B136 (1984) 243; B138 (1984) 293; A. Parkes and P. West, Phys. Lett. B138 (1984) 99; B127 (1983) 353; J.-M. Frere, L. Mezinescu and J.-P. Yao, Phys. Rev. D29 (1984) 1196; S. Hamidi and J.H. Schwarz, Phys. Lett. B147 (1984) 301; S. Rajpoot and J.G. Taylor, Phys. Lett. B147 (1984) 91; J.-P. Derendinger, S. Ferrara and A. Masiero, Phys. Lett. B143 (1984) 133; S. Kalara, D. Chang, R.N. Mohapatra and A. Gangopadhyay, Phys. Lett. B145 (1984) 323.

[3] P. Fayet, Phys. Lett. B153 (1985) 397; J.E. Bjorkman, D.R.T. Jones and S. Raby, Nucl. Phys. B259 (1985) 503; O. Piguet and K. Sibold, Phys. Lett. B177 (1986) 373.

[4] M. Böhm and A. Denner, Nucl. Phys. B282 (1987) 206.

[5] I.L. Shapiro and E.G. Yagunov, Int. J. Mod. Phys. A8 (1993) 1787; W. Lucha and F.F. Schöberl, preprint UWThPh-1993-56 (1993).

[6] P.S. Howe, K.S. Stelle and P.K. Townsend, Nucl. Phys. B214 (1983) 519; B236 (1984) 125.

[7] S.D. Odintsov and I.L. Shapiro, JETP Lett. 49 (1989) 125; Mod. Phys. Lett. A4 (1989) 1479.

[8] A.V. Ermushev, D.I. Kazakov and O.V. Tarasov, Nucl. Phys. B281 (1987) 72; D. Kapetanakis, M. Mondragón and G. Zoupanos, Z. Phys. C60 (1993) 181.

[9] I.L. Buchbinder, S.D. Odintsov and I.M. Lichtzier, Class. Quant. Grav. 6 (1989) 6055.

[10] I.L. Buchbinder, S.D. Odintsov and I.L. Shapiro, Effective Action in Quantum Gravity (IOP Publishing, Bristol and Philadelphia, 1992).

[11] S. Coleman and E. Weinberg, Phys. Rev. D7 (1973) 888.

[12] C. Ford, D.R.T. Jones, P.W. Stephenson and M.B. Einhorn, Nucl. Phys. B395 (1993) 17.

[13] E. Elizalde and S.D. Odintsov, Phys. Lett. B303 (1993) 240. 
[14] G.M. Shore, Ann. Phys. (NY) 128 (1980) 376.

[15] S.D. Odintsov and F.Sh. Zaripov, Mod. Phys. Lett. A4 (1989) 1955.

[16] D. Salopek, J.R. Bond and J.M. Bardeen, Phys. Rev. D40 (1989) 1753.

[17] E.W. Kolb and M.S. Turner, The Early Universe (Addison-Wesley, Reading, MA, 1990).

[18] I.L. Shapiro, preprint HUPD-9405 (1994).

[19] M. Bando, T. Kugo, N. Maekawa and H. Nakano, Prog. Theor. Phys. 90 (1993) 405.

[20] E. Elizalde and S.D. Odintsov, Phys. Lett. B321 (1994) 199.

[21] S. Deser, R. Jackiw and S. Templeton, Ann. Phys. (NY) 140 (1982) 372.

[22] A. Niemi and G. Semenoff, Phys. Rep. 135 (1987) 3.

[23] L.V. Avdeev, D.I. Kazakov and G.V. Grigoryev, preprint CERN-6091 (1991).

[24] S.D. Odintsov, Z. Phys. C54 (1992) 527. 